\title{
Mapping Electronic State Changes with STEM EBIC
}

B. C. Regan ${ }^{1}$, Toyanath Joshi ${ }^{2}$, Jared J. Lodico ${ }^{1}$, Brian T. Zutter ${ }^{1}$, Ho Leung Chan ${ }^{1}$, Matthew Mecklenburg $^{3}$, David Lederman ${ }^{2}$, and William A. Hubbard ${ }^{1}$

1. Department of Physics \& Astronomy and California NanoSystems Institute, University of California, Los Angeles, CA, USA.

2. Department of Physics, University of California, Santa Cruz, CA, USA.

3. Core Center of Excellence in Nano Imaging, University of Southern California, Los Angeles, USA.

As an imaging technique, transmission electron microscopy (TEM) is distinguished by its ability to precisely locate the positions of individual atoms. In many cases, it can even identify these atoms' elemental identities. However, while in principle such location and identification determines nearly all the properties of a sample, and in practice it serves as the key input to calculations (e.g. density functional theory) that can deduce these properties, standard TEM by itself is fundamentally blind to many properties of interest. For instance, standard TEM imaging does not distinguish between an insulator and a conductor, a distinction that can be made with the naked eye (at much lower resolution, of course).

In nanoelectronic devices, conductivity is a determiner of device function. To investigate such functioncritical properties with the high-resolution of TEM, we are developing scanning TEM electron beam induced current (STEM EBIC) imaging [1,2]. In STEM EBIC imaging, as the focused electron beam is rastered over the sample, the current induced in the sample is measured and associated with the beam position to produce an image. In the simplest case the current might be collected from the entire sample via a standard metal TEM sample grid, for example. If the sample is a device, however, there are often multiple leads from which EBICs can be collected and measured, which allows the possibility of differential contrast [2].

To produce a switchable electronic device for STEM EBIC imaging, we have fabricated electrontransparent, two-terminal devices that incorporate $\mathrm{NbO}_{2}$ as their active element (Fig. 1, top). Niobia exhibits an insulator-to-metal transition as its temperature is increased past $1070 \mathrm{~K}$ [3], and a hysteretic current-voltage characteristic when subjected to a strong electrical bias [4]. The relationship between the temperature-induced and the bias-induced transitions is poorly understood at present. Because the transitions occur between radically different electronic states (i.e. between an insulator and a metal), $\mathrm{NbO}_{2}$ is an ideal subject for demonstrating the revelatory powers of STEM EBIC imaging.

E-beam lithography is used to form Ti/Pt $(5 / 25 \mathrm{~nm})$ probe electrodes on a 20 -nm-thick silicon nitride window in a $200-\mu \mathrm{m}$ thick silicon chip. A 30-nm-thick layer of niobia is then deposited using pulsedlaser-deposition (PLD) [4] and capped with $20 \mathrm{~nm}$ of alumina via atomic-layer-deposition (ALD). Finally, the device is coated with a $30 \mathrm{~nm}$ layer of amorphous carbon. In this architecture the device itself consists of the metal electrodes and the $\mathrm{NbO}_{2}$, while the carbon top electrode is employed to provide diagnostic information about the device via STEM EBIC imaging.

Collecting the STEM EBIC from the carbon top electrode (Fig. 1 top) allows us to visualize electronic state changes in the device. As the bias voltage between the probe electrodes is increased from $0 \mathrm{~V}$ to 1 $\mathrm{V}$ to $1.8 \mathrm{~V}$, the BF and ADF STEM images show no changes (Fig. 1 bottom): standard STEM is 
insensitive to changes in potential. STEM EBIC, on the other hand, reveals dramatic changes. In standard EBIC imaging, current is generated by electron hole-pair separation in the presence of an electric field that is typically intrinsic to the device itself [1]. The architecture used here creates a vertical electric field between the carbon top electrode (held at virtual ground) and any part of the device below that is at a non-zero potential. Electron-hole pair creation in the alumina produces an EBIC that is proportional to the electric field. Since the alumina is deposited via conformal ALD, the distance between the device and top electrode is constant. Thus the electric field map provided by EBIC imaging is equivalent to an electrical potential map.

EBIC maps (Fig. 1) clearly reveal the large potential drop between the two probes at $1 \mathrm{~V}$ applied bias and the (perhaps surprising) smaller potential drop at the larger 1.8V applied bias. In the low resistance state (LRS) at $1.8 \mathrm{~V}$, finite lead resistance and sizable bias currents combine to cause much of the potential drop to occur outside the field of view. Linear combinations of maps [2] acquired with opposite bias polarities separate, for example, the signals due to internal device fields from those due to conductivity or temperature changes, revealing further details relevant to actual device function that cannot be detected with standard TEM [5].

\section{References:}

[1] ER White et al., Applied Physics Letters 107 (2015) 223104.

[2] WA Hubbard et al., Physical Review Applied 10 (2018) 044066.

[3] RF Janninck and DH Whitmore, Journal of Physics and Chemistry of Solids 27 (1966), p. 1183.

[4] T Joshi et al, Journal of Physics D: Applied Physics 48 (2015), 335308.

[5] This work was supported by National Science Foundation (NSF) award DMR-1611036, by NSF

Science and Technology Center (STC) award DMR-1548924 (STROBE), and by the UCLA PSEIF.
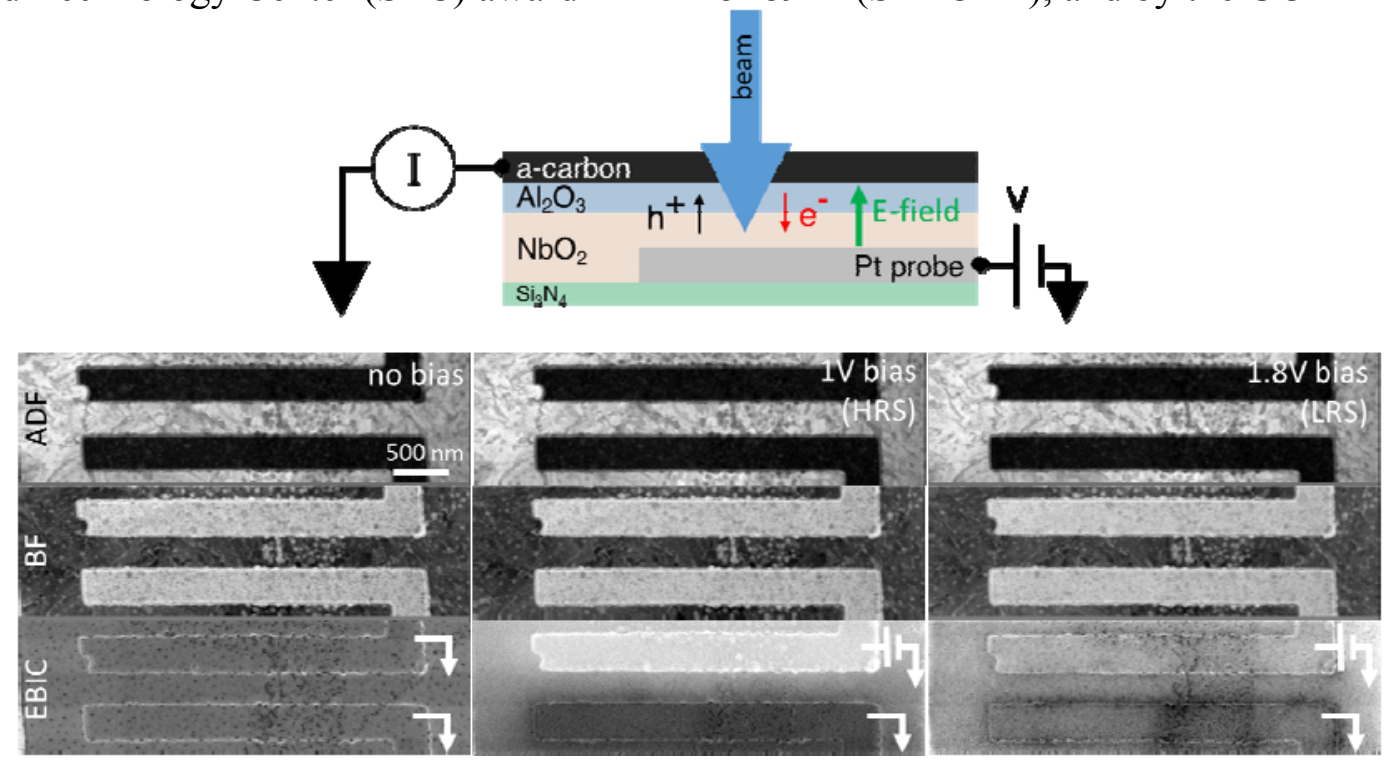

Figure 1: (top) Cartoon showing $\mathrm{NbO}_{2}$ devices with carbon top-electrode in cross section. (bottom) Matrix of STEM images acquired under various device bias conditions. The three rows show STEM bright field (BF), annular dark field (ADF), and EBIC (EBIC) images respectively, while the columns show, respectively, zero bias, $1 \mathrm{~V}$ bias with the $\mathrm{NbO}_{2}$ still in the high resistance state (HRS), and 1.8V with the $\mathrm{NbO}_{2}$ transitioned to the low resistance state (LRS). The EBIC images are all set to the same contrast scale. 